\title{
Extensive bilateral diffuse infiltrates
} and deterioration of lung following infection with severe acute respiratory syndrome coronavirus 2 in a pregnant woman: a case report

\author{
Somayeh Moeindarbary ${ }^{*}$, Salmeh Dadgar ${ }^{1}$, Parvaneh Layegh ${ }^{2}$, Zahra Shahriari ${ }^{3}$, Faezeh Fayyaz ${ }^{4}$,
} Sina Danesteh ${ }^{3}$, Mahdi Rafiee ${ }^{3}$ and Milad Bahrami ${ }^{4}$

\begin{abstract}
Introduction: Severe acute respiratory syndrome coronavirus 2 is the third member of the coronavirus family to cause global concern in the twenty-first century. Pregnant women are particularly at higher risk of developing severe viral pneumonia, possibly because of a partial immune suppression during their pregnancy. Under such critical and rapidly evolving circumstances, these poor findings might be helpful for the treatment of infected pregnant women with the 2019 novel coronavirus.

Case presentation: In this study, we report the case of a 33-year-old Asian pregnant woman at 25 gestational weeks with coronavirus disease 2019 who developed severe complications, including hypoxemia, acute respiratory distress syndrome, pulmonary infiltration, and bilateral pleural effusion. She died 1 month after admission to the hospital.

Conclusion: Pregnant populations are especially at higher risk of viral pneumonia development caused by severe acute respiratory syndrome coronavirus 2. Further research on the prevention and treatment of the new coronavirus is necessary.
\end{abstract}

Keywords: Maternal death, SARS-CoV-2, Pulmonary fibrosis, Case report, COVID-19

\section{Introduction}

Severe acute respiratory syndrome coronavirus 2 (SARS$\mathrm{CoV}-2)$ is the novel coronavirus that was first discovered in the Chinese city Wuhan in December 2019 [1]. The outbreak of the SARS-CoV-2) was declared a global pandemic by the World Health Organization (WHO) on 11 March 2020. To date, there have been approximately 5 million confirmed cases, resulting in thousands of

\section{*Correspondence: moeins@mums.ac.ir}

${ }^{1}$ Department of Obstetrics and Gynecology, Neonatal and Maternal Research Center, Mashhad University of Medical, Mashhad, Iran

Full list of author information is available at the end of the article deaths worldwide [2]. The severity of symptoms ranges from mild symptoms to severe illness. The most frequent symptoms include cough, pharyngitis, fever, myalgia, chills, and dyspnea [3].

Severe dyspnea is caused by the progression of lung lesions in multiple lung lobes, and in some cases in advanced stages, white lung syndrome is observed. Lung lesions are manifested as airspace opacities on plain chest radiography, or ground-glass opacities or consolidation on chest radiography images, usually with a rounded morphology and a peripheral lung distribution, which is a diagnostic method for coronavirus disease 2019 permits use, sharing, adaptation, distribution and reproduction in any medium or format, as long as you give appropriate credit to the original author(s) and the source, provide a link to the Creative Commons licence, and indicate if changes were made. The images or other third party material in this article are included in the article's Creative Commons licence, unless indicated otherwise in a credit line to the material. If material is not included in the article's Creative Commons licence and your intended use is not permitted by statutory regulation or exceeds the permitted use, you will need to obtain permission directly from the copyright holder. To view a copy of this licence, visit http://creativecommons.org/licenses/by/4.0/. The Creative Commons Public Domain Dedication waiver (http://creativeco mmons.org/publicdomain/zero/1.0/) applies to the data made available in this article, unless otherwise stated in a credit line to the data. 
(COVID-19) [4-6]. Pregnant women are particularly at higher risk of developing severe viral pneumonia, possibly because of a partial immune suppression during their pregnancy [7]. Pneumonia was reported as the third most common cause of mortality among pregnant women [8]. Previous data on Middle East respiratory syndrome (MERS) and severe acute respiratory syndrome (SARS) suggest that adverse pregnancy outcomes may occur due to the infection [9]. According to some studies, COVID19 in the pregnant population can cause premature delivery and intrauterine growth restriction [6].

The effect of COVID-19 on pregnancy outcomes is not clear yet. Moreover, there has been no consensus on the maternal-fetal transmission of SARS-CoV-2 to date. Also, it has not been well understood whether preterm delivery ameliorates the symptoms of a critically ill mother. In this case report, we present the case of a 33-year-old Asian pregnant woman who was infected with SARS-CoV-2 in the late second trimester of pregnancy. Under such critical and rapidly evolving circumstances, these poor findings might be helpful for the treatment of pregnant women who are infected with SARS-CoV-2 in Iran and elsewhere.

\section{Case presentation}

A 33-year-old Asian pregnant woman para 2, gravida 3, gestational age of 25 weeks, was hospitalized with fever, chills, shortness of breath, and myalgia. She had her first baby delivered by vaginal delivery 5 years ago and her second by cesarean section 2 years ago. The patient had no underlying diseases and did not have direct contact with COVID-19 cases. Vital signs of the patient were recorded as follows: respiratory rate (RR) 26 breaths per minute, blood pressure (BP) $110 / 70 \mathrm{mmHg}$, temperature (T) $39.5{ }^{\circ} \mathrm{C}$, heart rate (HR) 110 beats per minute, and oxygen saturation $\left(\mathrm{SpO}_{2}\right) 94 \%$. The laboratory results showed that lymphocyte count was lower than normal (lymphocyte count $1 \times 10^{9} / \mathrm{L}$ ). While platelet count, hepatic enzymes, and creatinine levels were within the normal range, C-reactive protein level (CRP) was significantly increased. Coagulation function and blood biochemistry were normal (Table1). A GeneXpert SARS-CoV-2 RNA polymerase chain reaction (PCR) test was performed, and the result was positive. The patient had no complaints about uterine contractions, hemorrhage, and symptoms of amniorrhexis. Bilateral involvement was detected on chest computed tomography $(\mathrm{CT})$, and on lung auscultation, diminished breath sound was detected. A day after the hospitalization, the patient was transferred to the intensive care unit (ICU) because of the shortness of breath (RR 32 breaths per minute) and low oxygen saturation $\left(\mathrm{SpO}_{2} 88 \%\right)$. CT chest on the second day of hospitalization revealed an exacerbation of pulmonary involvement. The patient was intubated 1 week after hospitalization owing to a reduction in the oxygen saturation to $80 \%$ and exacerbation of respiratory distress. Chest CT on the eighth day of hospitalization showed extensive bilateral diffuse infiltrates and deterioration of lung involvement (Fig. 1). From the beginning of hospitalization, despite the use of broad-spectrum antibiotic coverage, the patient's fever was intermittent.

One week after transferring to the ICU and due to the patient's deteriorating condition, plasmapheresis was performed twice with 10 units of fresh frozen plasma (FFP) for the patient, which did not improve her condition. Also, due to a decrease in hemoglobin (HB $8.1 \mathrm{~g} / \mathrm{dl})$,

Table 1 Laboratory characteristics by the day of hospitalization

\begin{tabular}{|c|c|c|c|c|c|c|c|c|}
\hline & Day 1 & Day 4 & Day 8 & Day 12 & Day 16 & Day 20 & Day 24 & Day 28 \\
\hline White blood cell count, $\times 10^{9} / \mathrm{L}$ & 10.1 & 9.2 & 8.1 & 7.3 & 9.0 & 8.3 & 15.2 & 20.5 \\
\hline Lymphocyte count, $\times 10^{9} / \mathrm{L}$ & 1.0 & 1.0 & 1.0 & 0.8 & 0.8 & 0.5 & 0.7 & 0.5 \\
\hline Hemoglobin, g/L & 12.8 & 11 & 8.8 & 9.6 & 9.4 & 8.3 & 11 & 10.5 \\
\hline Platelet count, $\times 10^{9} / \mathrm{L}$ & 310 & 345 & 250 & 210 & 158 & 140 & 144 & 126 \\
\hline C-reactive protein, mg/L & 79 & 50 & & & & & & \\
\hline Alanine aminotransferase, U/L & 15 & & 24 & & 28 & & & 35 \\
\hline Aspartate aminotransferase, U/L & 25 & & 30 & & 30 & & & 32 \\
\hline Creatinine, $\mu \mathrm{mol} / \mathrm{L}$ & 0.6 & 0.8 & 0.9 & 1 & 1 & 1.1 & 1.5 & 1.5 \\
\hline \multicolumn{9}{|l|}{ VBG } \\
\hline $\mathrm{pH}$ & 7.39 & 7.46 & 7.41 & 7.49 & 7.39 & 7.42 & 7.19 & \\
\hline $\mathrm{PO}_{2}, \mathrm{mmHg}$ & 59.5 & 42 & 40 & 35.7 & 74.9 & 38 & 43.7 & \\
\hline $\mathrm{PCO}_{2}, \mathrm{mmHg}$ & 74.6 & 41.8 & 42.3 & 46.7 & 48 & 43.4 & 68.8 & \\
\hline $\mathrm{HCO}_{3}, \mathrm{mEq} / \mathrm{L}$ & 45.1 & 30.1 & 30.5 & 31.5 & 29.5 & 28.2 & 26.2 & \\
\hline $\mathrm{BE}, \mu \mathrm{mol} / \mathrm{L}$ & 17.5 & 6.7 & 8.1 & 12.5 & 4.6 & 3.1 & 43.4 & \\
\hline $\mathrm{O}_{2}$ Sat, $\%$ & 90.4 & 80.5 & 82 & 80 & 94.9 & $72.8 \%$ & 65.4 & \\
\hline
\end{tabular}




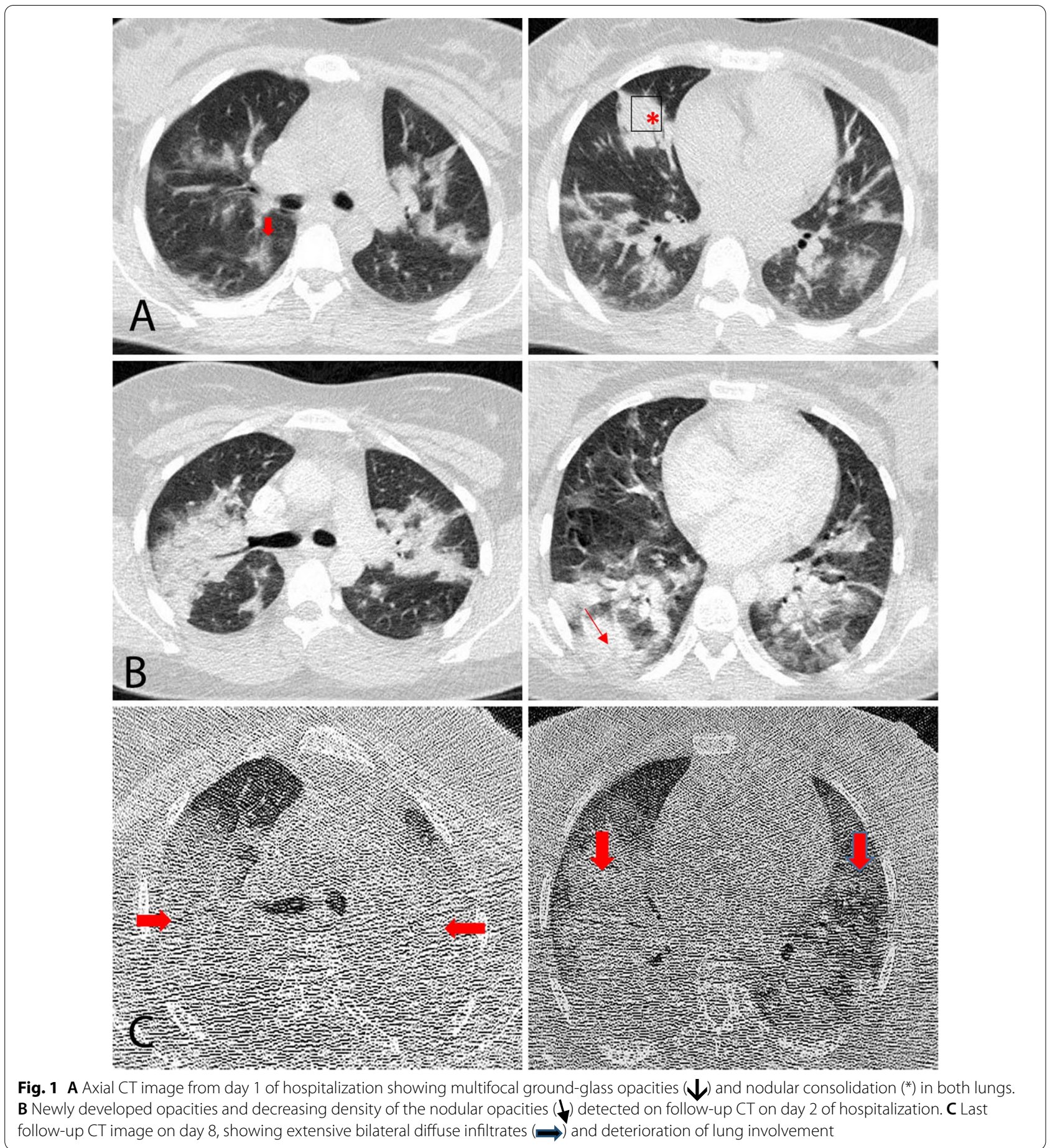

two units of packed cells were transfused twice. Three weeks after hospitalization, ultrasounds were performed several times to assess fetal growth, and a sharp decrease in the amniotic fluid (amniotic fluid index $2.5 \mathrm{~cm}$ ) and fetal growth retardation were observed. To rule out the possibility of a preterm ruptured membrane, an
AmniSure test was performed twice, which was negative both times. Due to the condition of the fetus and the exacerbation of the patient's respiratory condition, it was decided in the committee constituting of specialists in the special care and departments of lung and perinatology that the pregnancy should be terminated to 
improve the patient's respiratory condition. Therefore, to induce cervical ripening, an intracervical Foley catheter was placed and extra-amniotic saline infusion was performed. After receiving three doses of vaginal misoprostol at doses of 50, 100, and $200 \mu \mathrm{g}$ every 6 hours, a $600 \mathrm{~g}$ baby was born with a 1-minute Apgar score of 2 and a 5-minute Apgar score of 4, and the fetal heart rate was 145 beats per minute. The newborn was intubated and transferred to the neonatal intensive care unit (NICU) and died after 12 hours.

One week after the termination of pregnancy, due to the observation of bilateral pleural effusion on the chest CT, a chest tube was installed on both sides. Since no improvement in lung condition was observed and complete destruction of lung tissue was detected, the patient died 1 month after hospitalization.

\section{Discussion and conclusion}

Lessons learned from the previous viral outbreaks have proven the negative impacts of viral infection on maternal, neonatal, and fetal outcomes. For instance, the total mortality rate of 1918 influenza was $2.6 \%$, while it was $37 \%$ among pregnant women [10]. It was shown that MERS or SARS infection during pregnancy correlated with a higher incidence of severe maternal and fetal complications, such as admission to the ICU, preterm delivery, maternal and fetal mortality, and intrauterine growth restriction [11]. According to a study conducted by Nan $\mathrm{Yu}$ and associates, the pregnancy outcomes of pregnant women with COVID-19 are considerably better than those of pregnant women with SARS [12].

As previously discussed, pregnant women are especially susceptible to the adverse complications of viral pneumonia. However, for COVID-19, data regarding the differences in the clinical features of pregnant and nonpregnant women are yet limited. Chen et al. have reported that the clinical signs and symptoms of pregnant women with COVID-19 are similar to those of nonpregnant women [13]. Also, in a large case series conducted in the Hubei Province of China, the incidence of severe pneumonia in the pregnant population was the same as in the general population [14]. More clinical evidence is needed to support this relationship.

Fever is the most prevalent symptom in pregnant women infected with SARS-CoV-2, observed in $78 \%$ of the cases [15]. Consistent with previous studies, our patient presented with fever, chills, dyspnea, and myalgia. It is confirmed that COVID-19 is associated with a decrease in lymphocytes, as observed in our study [13]. One study performed chest CT scans on 15 pregnant women with COVID-19 and indicated that the most common early finding was ground-glass opacities, as seen in our case [15]. Many studies have reported that adverse outcomes and mortality are more likely to occur in patients with underlying medical conditions, whereas the present case did not have any underlying medical condition. In our study, the administration of convalescent plasma did not improve the clinical status of the patient, similar to the study of Niveditha that demonstrated that convalescent plasma has few or no clinical benefits for the treatment of COVID-19 [16]. It is not clear yet whether convalescent plasma with specific antibodies against SARS-CoV-2 that is collected from patients recovered from COVID-19 can improve the survival of critically ill patients. However, the plasma therapy has not been approved yet for use by the United States Food and Drug Administration, and numerous clinical trials are working on it [17].

Maternal complications of the present case were also in line with previous reports, which have found that pregnant women with severe or critical COVID-19 have a higher chance of developing respiratory failure, using mechanical ventilation, and maternal death, as well as fetal complications including preterm birth, intrauterine growth restriction, and intrauterine fetal death [18-21]. On the contrary, Zaigham et al. conducted a systematic review and, surprisingly, have suggested a lower rate of admission to the ICU, no maternal deaths, and only one neonatal death and one intrauterine fetal death [22].

In this patient, due to lack of improvement in respiratory status and progressive course of pulmonary destruction, as well as lack of fetal growth within 3 weeks and decreased immunity, it was decided to terminate the pregnancy to improve the respiratory condition. This is consistent with the rising concern that Castro et al. reported regarding preterm delivery in pregnant women with COVID-19. In their study, they found that $41 \%$ of deliveries in pregnant women with COVID-19 occurred before 37 weeks of pregnancy [23].

The limitation of our study was that no real-time polymerase chain reaction test was conducted on the premature infant, so we do not have any information about vertical transmission.

Since it has been proven in numerous studies that the pregnant population is especially at higher risk of viral pneumonia caused by coronaviruses, further research on the prevention and treatment of the new coronavirus is necessary.

\section{Abbreviations}

COVID-19: Coronavirus disease 2019; WHO: World Health Organization; ARDS: Acute respiratory distress syndrome; SARS-CoV-2: Severe acute respiratory syndrome coronavirus 2; RT-PCR: Real-time polymerase chain reaction; CT: Computed tomography; BP: Blood pressure; HR: Heart rate; GGO: Groundglass opacity; T: Temperature; RR: Respiratory rate; CRP: C-reactive protein; ICU: Intensive care unit; $\mathrm{SpO}_{2}$ : Oxygen saturation; FFP: Fresh frozen plasma; $\mathrm{NICU}$ : 
Neonatal intensive care unit; ABG: Arterial blood gas; BE: Base excess; HB: Hemoglobin.

\section{Acknowledgements}

We would like to thank the patient's family for their cooperation. Also, we wish to express our gratitude to the nurses who took care of the patient presented in this case report.

\section{Authors' contributions}

SD and PL conceived the idea. SM collected patient data and described it in the case report with a literature review. ZSh, FF, MB, SD, and MR had the primary responsibility for the writing of the manuscript. All authors read and approved the final manuscript.

\section{Funding}

This study was supported by the Student Research Committee of Mashhad University of Medical Sciences in Iran.

\section{Availability of data and materials}

The patient's information and medical records used for the case report are available from the corresponding author upon request.

\section{Declarations}

\section{Ethics approval and consent to participate}

This study did not include experiments on animals or humans. The patient's next of kin consented to the use of her personal data for the purpose of this case report.

\section{Consent for publication}

Written informed consent was obtained from the patient's next of kin for publication of this case report and any accompanying images. A copy of the written consent is available for review by the Editor-in-Chief of this journal.

\section{Competing interests}

The authors declare that they have no competing interests.

\section{Author details}

'Department of Obstetrics and Gynecology, Neonatal and Maternal Research Center, Mashhad University of Medical, Mashhad, Iran. ${ }^{2}$ Department of Radiology, School of Medicine, Mashhad University of Medical Sciences, Mashhad, Iran. ${ }^{3}$ Student Research Committee, Faculty of Medicine, Mashhad University of Medical Sciences, Mashhad, Iran. ${ }^{4}$ Student Research Committee, Faculty of Paramedical Sciences, Mashhad University of Medical Sciences, Mashhad, Iran.

Received: 3 June 2020 Accepted: 18 October 2021

Published online: 13 December 2021

\section{References}

1. Zhu N, Zhang D, Wang W, Li X, Yang B, Song J, et al. A novel coronavirus from patients with pneumonia in China, 2019. N Engl J Med. 2020;382(8):727-33.

2. World Health Organization. https://covid19.who.int/.

3. Zhong K, Wang RX, Qian XD, Yu P, Zhu XY, Zhang Q, et al. Neuroprotective effects of saffron on the late cerebral ischemia injury through inhibiting astrogliosis and glial scar formation in rats. Biomed Pharmacother. 2020;126:110041.

4. Huang C, Wang Y, Li X, Ren L, Zhao J, Hu Y, et al. Clinical features of patients infected with 2019 novel coronavirus in Wuhan, China. Lancet. 2020;395(10223):497-506.

5. Nie S, Han S, Ouyang H, Zhang Z. Coronavirus disease 2019-related dyspnea cases difficult to interpret using chest computed tomography. Respir Med. 2020;167:105951.

6. Dashraath P, Wong JLJ, Lim MXK, Lim LM, Li S, Biswas A, et al. Coronavirus disease 2019 (COVID-19) pandemic and pregnancy. Am J Obstet Gynecol. 2020;222:521.
7. Kareva I. Immune suppression in pregnancy and cancer: parallels and insights. Transl Oncol. 2020;13(7):100759.

8. Visscher HC, Visscher RD. Indirect obstetric deaths in the state of Michigan 1960-1968. Am J Obstet Gynecol. 1971;109(8):1187-96.

9. Schwartz DA, Graham AL. Potential maternal and infant outcomes from (Wuhan) coronavirus 2019-nCoV infecting pregnant women: lessons from SARS, MERS, and other human coronavirus infections. Viruses. 2020;12(2):194.

10. Gottfredsson M. [The Spanish flu in Iceland 1918. Lessons in medicine and history]. Laeknabladid. 2008;94(11):737-45.

11. Schwartz DA, Graham AL. Potential maternal and infant outcomes from coronavirus 2019-nCoV (SARS-CoV-2) infecting pregnant women: lessons from SARS, MERS, and other human coronavirus infections. Viruses. 2020;12(2):194.

12. Yu N, Li W, Kang Q, Xiong Z, Wang S, Lin X, et al. Clinical features and obstetric and neonatal outcomes of pregnant patients with COVID-19 in Wuhan, China: a retrospective, single-centre, descriptive study. Lancet Infect Dis. 2020;20(5):559-64.

13. Chen H, Guo J, Wang C, Luo F, Yu X, Zhang W, et al. Clinical characteristics and intrauterine vertical transmission potential of COVID-19 infection in nine pregnant women: a retrospective review of medical records. Lancet. 2020;395(10226):809-15.

14. Yan J, Guo J, Fan C, Juan J, Yu X, Li J, et al. Coronavirus disease 2019 in pregnant women: a report based on 116 cases. Am J Obstet Gynecol. 2020;223:111.

15. Liu D, Li L, Wu X, Zheng D, Wang J, Yang L, et al. Pregnancy and perinatal outcomes of women with coronavirus disease (COVID-19) pneumonia: a preliminary analysis. AJR Am J Roentgenol. 2020;215(1):127-32.

16. Devasenapathy N, Ye Z, Loeb M, Fang F, Najafabadi BT, Xiao Y, et al. Efficacy and safety of convalescent plasma for severe COVID-19 based on evidence in other severe respiratory viral infections: a systematic review and meta-analysis. CMAJ. 2020;197:E745-55.

17. Sadeghnia HR, Shaterzadeh H, Forouzanfar F, Hosseinzadeh H. Neuroprotective effect of safranal, an active ingredient of Crocus sativus, in a rat model of transient cerebral ischemia. Folia Neuropathol. 2017;55(3):206-13.

18. Alzamora MC, Paredes T, Caceres D, Webb CM, Valdez LM, La Rosa M. Severe COVID-19 during pregnancy and possible vertical transmission. Am J Perinatol. 2020;37:861-5.

19. Baud D, Greub G, Favre G, Gengler C, Jaton K, Dubruc E, et al. Secondtrimester miscarriage in a pregnant woman with SARS-CoV-2 infection. JAMA. 2020;323:2198.

20. Hantoushzadeh S, Shamshirsaz AA, Aleyasin A, Seferovic MD, Aski SK, Arian SE, et al. Maternal death due to COVID-19 disease. Am J Obstet Gynecol. 2020;223:109.e1.

21. Pierce-Williams RAM, Burd J, Felder L, Khoury R, Bernstein PS, Avila K, et al. Clinical course of severe and critical COVID-19 in hospitalized pregnancies: a US cohort study. Am J Obstet Gynecol MFM. 2020. https://doi.org/ 10.1016/j.ajogmf.2020.100134

22. Zaigham M, Andersson O. Maternal and perinatal outcomes with COVID19: a systematic review of 108 pregnancies. Acta Obstet Gynecol Scand. 2020;99:823-9.

23. Castro P, Matos AP, Werner H, Lopes FP, Tonni G, Araujo JE. Covid-19 and pregnancy: an overview. Rev Bras Ginecol Obstet. 2020;42(7):420-6.

\section{Publisher's Note}

Springer Nature remains neutral with regard to jurisdictional claims in published maps and institutional affiliations. 